\title{
2005 Cotton Variety Trials in Florida: Mid- and Late-Maturing Cultivars ${ }^{1}$
}

D. L. Wright, C. A. Smith, and E. B. Baxley²

Cotton variety trials were planted in conventional tilled soil with a cone planter at the seeding rate of 80 seeds/ 25 linear feet of row with 36" row spacing on May 18, 2005.

\section{Mid- and Late-Maturing Cotton Variety Trial}

Thirty-one mid- and late-maturing cotton varieties were planted in this trial on May 18, 2005. This followed a bedded application of $10 \mathrm{~N} 28 \mathrm{P} 87 \mathrm{~K}$ that was applied on March 21. The following is a summary of how the plots were managed throughout the 2005 growing season:

\section{3-21-05 Bedded 10N 28P 87K}

\section{5-18-05 Planted}

5-23-05 PRE Application - Prowl 1.5pt, Cotoran $1.5 \mathrm{pt}$, Glyphomax $2 \mathrm{qt}$,

Staple 8 oz, Orthene 4oz
6-27-05 200\#/Acre 3-7-28 50\# Kmag 50\# MOP (30 units) $70 \# \mathrm{~N}$ in irrigation

6-29-05 0.1oz/Acre Envoke

7-15-05 70\# N/Acre applied through irrigation.

7-20-05 4oz Bidrin 1\# Solubor 10oz Pix

7-27-05 1qt Caparol 1qt MSMA

8-13-05 16oz Pix 4oz Bidrin 4oz Ammo 2\# 20-20-20

\section{0-25-05 Harvest}

Cotton plots were harvested on October 25, 2005. These were spindle picked by a Case International 1822 two row cotton spindle harvester.

\section{Management Considerations for Maximum Yields}

1. Choosing the right variety: In 2005 there were 69 varieties; this is a lot of varieties to choose from. Special considerations should be made in regards to desired traits and your own farm.

1. This document is SS-AGR-34, one of a series of the Agronomy Department, Florida Cooperative Extension Service, Institute of Food and Agricultural Sciences, University of Florida. Original publication date April 2006. Visit the EDIS Web Site at http://edis.ifas.ufl.edu.

2. D. L. Wright, professor, Agronomy Department, North Florida Research and Education Center--Quincy, FL; C. A. Smith, former regional IPM agent, Jackson County, FL; ; E. B. Baxley, OPS Weed Scientist, North Florida Research and Education Center--Quincy, FL; Florida Cooperative Extension Service, Institute of Food and Agricultural Sciences, University of Florida, Gainesville, FL 32611.

The use of trade names in this publication is solely for the purpose of providing specific information. UF/IFAS does not guarantee or warranty the products named, and references to them in this publication does not signify our approval to the exclusion of other products of suitable composition.

The Institute of Food and Agricultural Sciences (IFAS) is an Equal Opportunity Institution authorized to provide research, educational information and other services only to individuals and institutions that function with non-discrimination with respect to race, creed, color, religion, age, disability, sex, sexual orientation, marital status, national origin, political opinions or affiliations. U.S. Department of Agriculture, Cooperative Extension Service, University of Florida, IFAS, Florida A. \& M. University Cooperative Extension Program, and Boards of County Commissioners Cooperating. Larry Arrington, Dean 
When making variety selections consider results from various locations from multiple years of University trials, and look for consistent performers.

2. Conservation Tillage: Strip-Till planting decreases erosion, increases soil organic matter, soil moisture, and improves soil texture which in turn may lead to increased yields. Cover crops should be killed 3 to 4 weeks prior to planting to reduce insect problems and prevent soil moisture depletion.

3. Planting dates: Optimum planting ranges from April 20 to May 20. Consider planting early and mid-full season varieties at the same time in order to stagger harvest dates.

4. Target Plant Population: Desired plant population is 2-3 plants per foot. In order to achieve this plant 3-4 seed per foot.

5. $\mathbf{N}$ fertilization: Both insufficient and excess nitrogen can lead to fruit shed or boll rot. Nitrogen should be applied near the row, early in the season at first squaring. Two applications may be made on sandy soils (at squaring and 3 weeks later). The recommended rate of nitrogen ranges from 50 to $90 \mathrm{lbs}$ N/A on most soils.

6. Weed control: Early season weed control is critical no matter what technology that you decide to use, and has proven to increase yields. Newer varieties that allow over the top herbicide applications all season long proved themselves as top yielders but still need to be treated for early season weeds, if applications are delayed until thresholds are maximized, the technology proves to be no advantage.

7. Insect Control: Effective scouting should be implemented with timely and proper insect management of bollworms, armyworms, and stinkbugs is critical.

\section{Timely defoliation and harvest: If the} defoliation is delayed until $60-65 \%$ of the total crop to be harvested is open, $90 \%$ of the crop can likely be harvested within two weeks after the application.

\section{Results}

Table 1 shows the harvest and the HVI data for the 2005 Mid-Late season cotton varieties. 


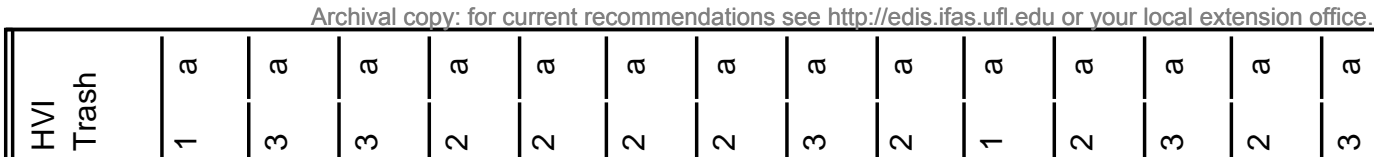

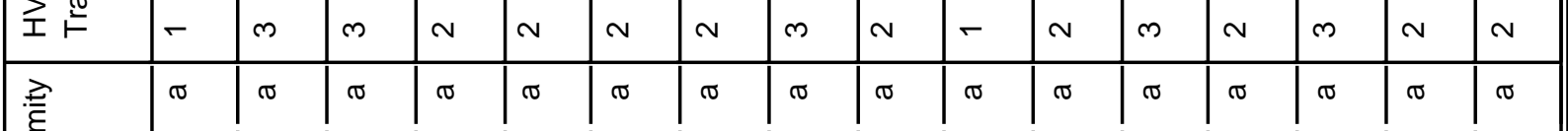

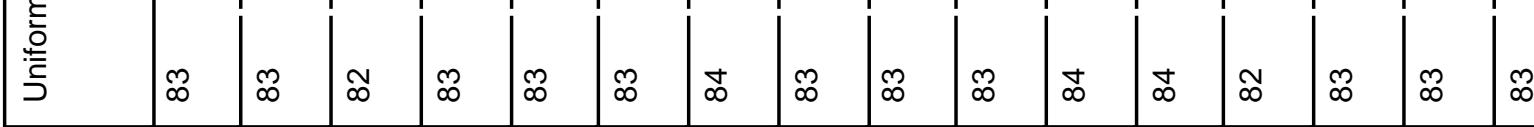

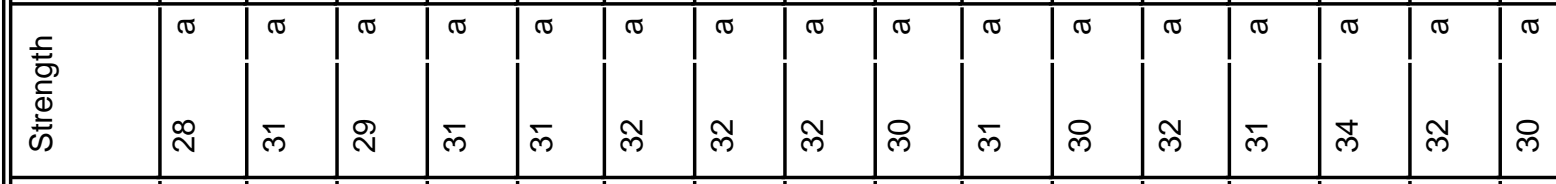

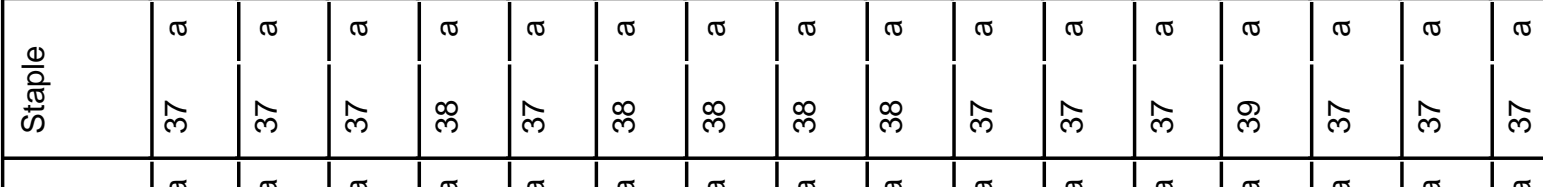

竞

旁

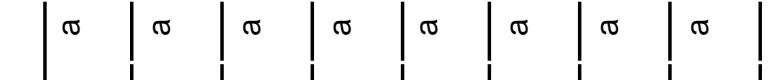

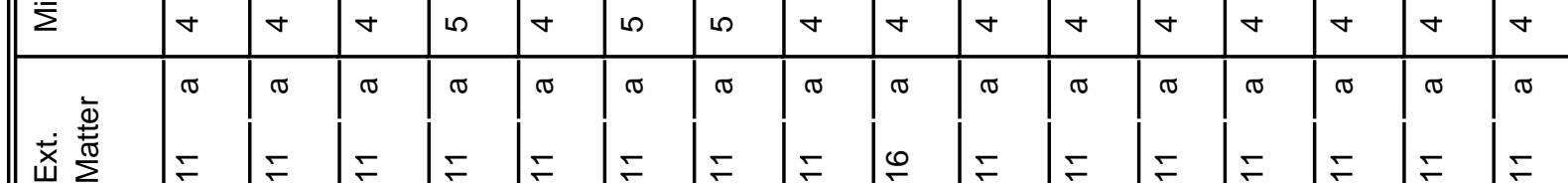

\begin{tabular}{|l|l|l|l|l|l|l|l|l|l|l|l|l|l|l|l|l} 
யे & 厂 & $\mp$ & $\mp$ & $\mp$ & $\mp$ & $\mp$ & $\mp$ & $\mp$ & 0 & $\mp$ & $\mp$ & $\mp$ & $\mp$ & $\mp$ & $\mp$ & $\mp$ \\
\hline
\end{tabular}

部

\begin{tabular}{|c|c|c|c|c|}
\hline$\tau$ & - & - & - & - \\
\hline$\widetilde{\sigma}$ & $\sigma$ & ఠర & ( & ఠ \\
\hline
\end{tabular}

\begin{tabular}{|c|c|c|c|c|c|c|c|c|c|c|c|c|c|c|c|c|}
\hline$\stackrel{0}{10}$ & $N$ & $\infty$ & $\infty$ & $\Lambda$ & $\infty$ & $\infty$ & $\infty$ & $\infty$ & $\infty$ & $\infty$ & $\infty$ & $\infty$ & $\infty$ & $\infty$ & $\infty$ & $\infty$ \\
\hline 흥 & $\sigma$ & $\widetilde{\sigma}$ & $\sigma$ & $\sigma$ & $\sigma$ & $\sigma$ & $\sigma$ & $\sigma$ & $\sigma$ & ఠ & $\sigma$ & $\widetilde{\sigma}$ & $\sigma$ & $\sigma$ & $\sigma$ & $\sigma$ \\
\hline
\end{tabular}

\begin{tabular}{|c|c|c|c|c|c|c|c|c|c|c|c|c|c|c|c|c|}
\hline & 几 & in & 点 & $\mathscr{q}$ & 夺 & g & $\mathscr{q}$ & 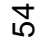 & 同 & g & โ5 & in & 5 & Lั & is & g \\
\hline & ๘ & बे & 요 & $\frac{0}{\pi}$ & $\frac{\circ}{\sigma}$ & $\frac{0}{\sigma}$ & $\frac{0}{\sigma}$ & $\frac{0}{\sigma}$ & $\frac{0}{\pi}$ & $\frac{0}{\pi}$ & $\frac{0}{\pi}$ & $\frac{0}{\pi}$ & बे & $\frac{0}{\sigma}$ & $\frac{0}{\pi}$ & \\
\hline
\end{tabular}

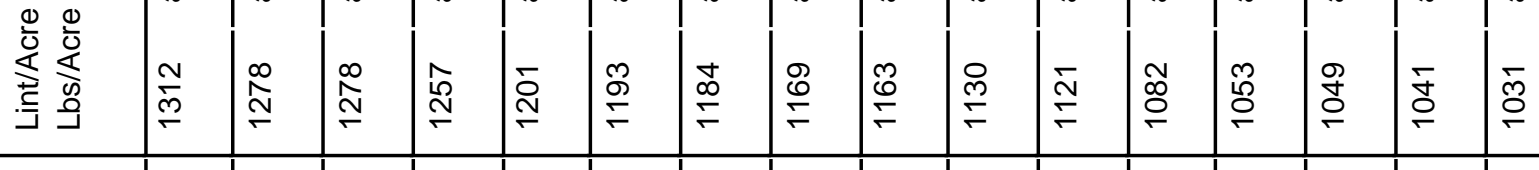

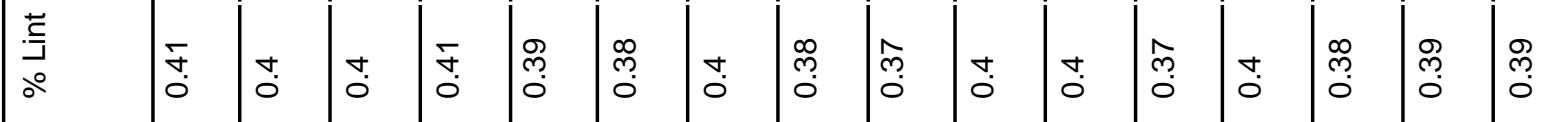

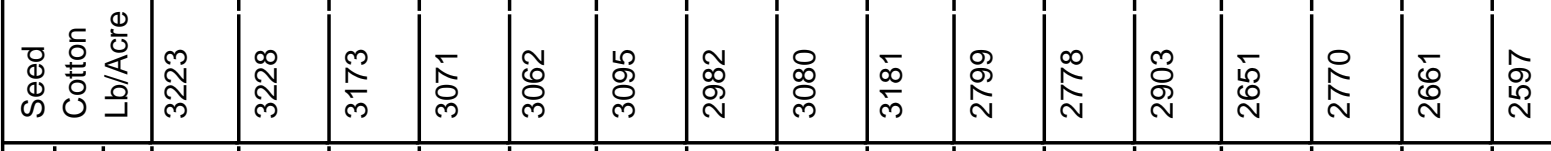

\begin{tabular}{|c|c|c|c|c|c|c|c|c|c|c|c|c|c|c|c|c|c|c|}
\hline $\mid \begin{array}{l}5 \\
0 \\
\overline{5} \\
\frac{1}{1} \\
\frac{1}{3} \\
\end{array}$ & 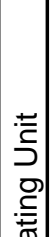 & 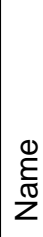 & 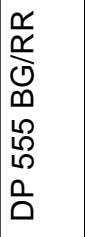 & 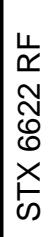 & 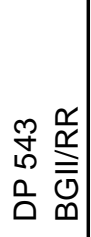 & 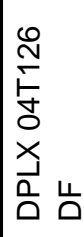 & \begin{tabular}{l}
\multicolumn{1}{c}{} \\
$\infty$ \\
$\infty$ \\
$\infty$ \\
$\infty$ \\
$⺊$ \\
$\omega$
\end{tabular} & 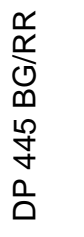 & 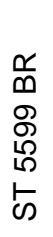 & $\begin{array}{l}\tilde{r} \\
\infty \\
0 \\
0 \\
\varnothing \\
6 \\
\omega\end{array}$ & 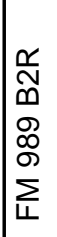 & 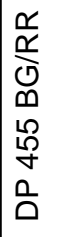 & 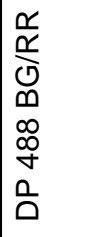 & 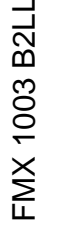 & 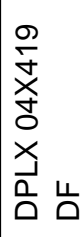 & 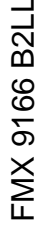 & 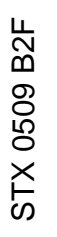 & 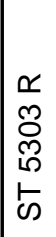 \\
\hline రి & & $\begin{array}{l}\frac{\bar{v}}{\pi} \\
\frac{\pi}{\omega} \\
\frac{\pi}{n}\end{array}$ & 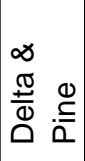 & 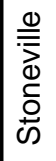 & 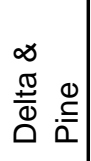 & 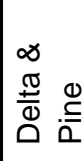 & 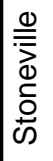 & 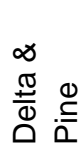 & 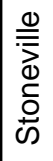 & 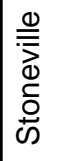 & 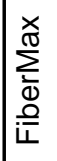 & 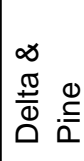 & 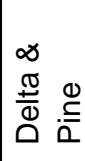 & 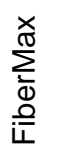 & 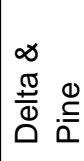 & 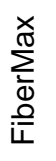 & 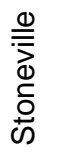 & 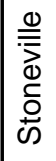 \\
\hline
\end{tabular}


Archival copy: for current recommendations see http:/ledis ifas ufl edu or vour local extension office.

\begin{tabular}{|c|c|c|c|c|c|c|c|c|c|c|c|c|c|c|c|}
\hline$=\frac{5}{9}$ & $\sigma$ & ช & రు & $\widetilde{\sigma}$ & $\sigma$ & 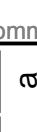 & ( & $\sigma$ & ס & uft.e & б & ఠ & ช & ๘ & $\sigma$ \\
\hline$\frac{\perp}{\text { エ }}$ & 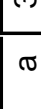 & \begin{tabular}{|l} 
\\
\end{tabular} & \begin{tabular}{|l} 
\\
\end{tabular} & 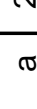 & 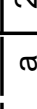 & \begin{tabular}{|l} 
\\
\end{tabular} & L & 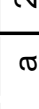 & 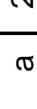 & 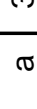 & 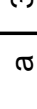 & 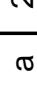 & 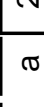 & 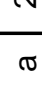 & 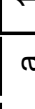 \\
\hline
\end{tabular}

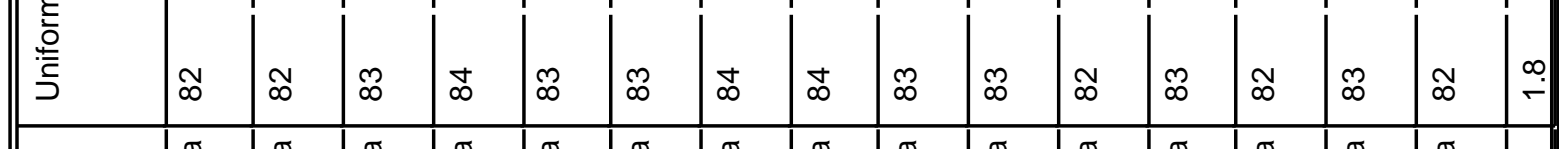

点

$\stackrel{5}{\stackrel{5}{\infty}}$

\begin{tabular}{|l|l|l|l|l|}
$\sigma$ & $\sigma$ & $\sigma$ & $\sigma$ & $\sigma$ \\
\hline
\end{tabular}

ह

$\frac{\frac{0}{0}}{\frac{\pi}{\infty}}$

$\frac{\sqrt{0}}{\frac{0}{20}}$

\begin{tabular}{|l|l|l|l|l}
$\sigma$ & $\sigma$ & $\sigma$ & $\sigma$ & $\sigma$
\end{tabular}

\begin{tabular}{|l|l|l|l|}
$\hat{m}$ & $\hat{m}$ & $\infty$ & $\infty$ \\
\hline
\end{tabular}

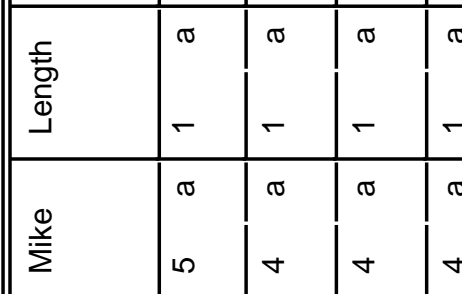

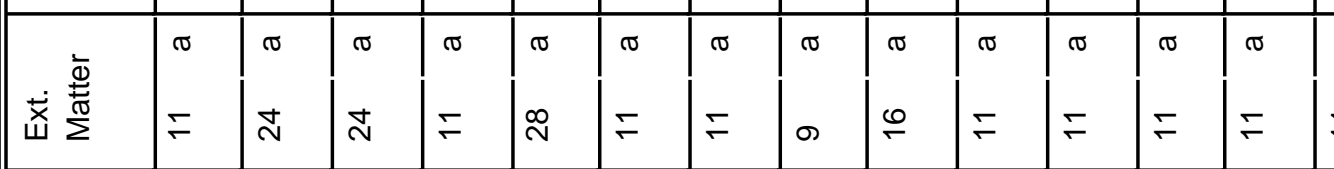

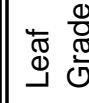

\begin{tabular}{|l|l|l|l|}
\hline & $\sigma$ & $\sigma$ &
\end{tabular}

更苋

흥 $\frac{\pi}{0} \frac{\pi}{\sigma}$

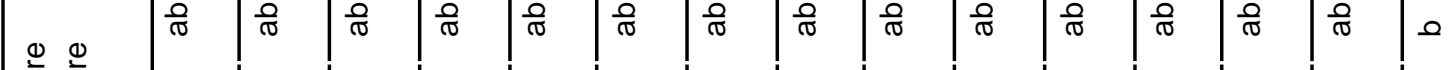

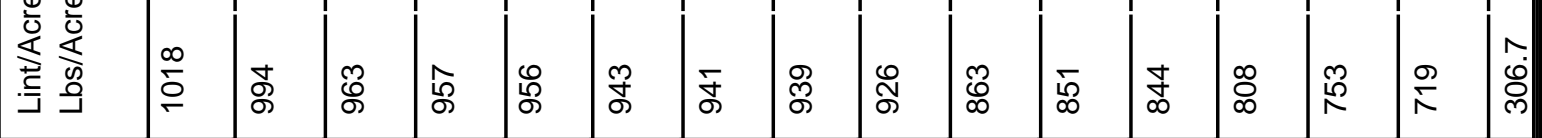

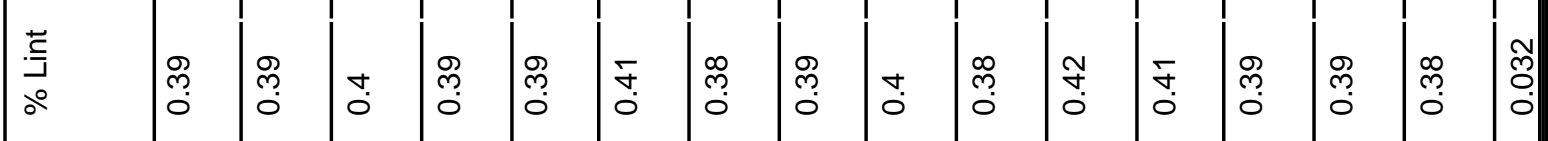

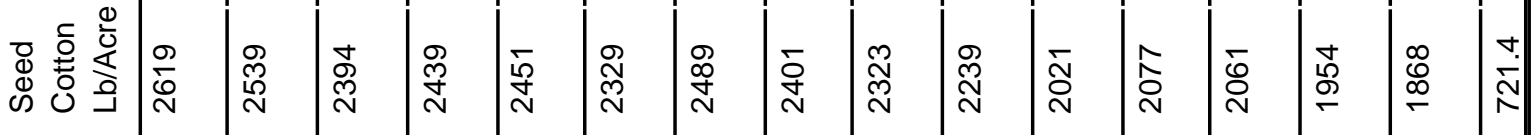

\begin{tabular}{|c|c|c|c|c|c|c|c|c|c|c|c|c|c|c|c|c|c|}
\hline $\begin{array}{l}\frac{1}{1} \\
\frac{1}{0}\end{array}$ & 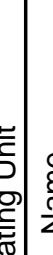 & 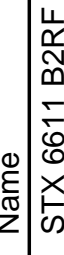 & 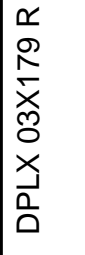 & 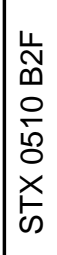 & 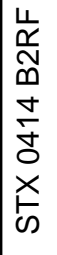 & 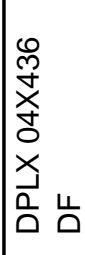 & 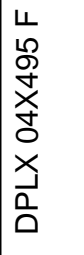 & 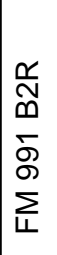 & 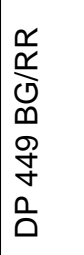 & 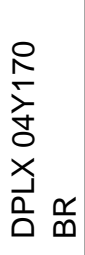 & 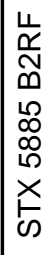 & 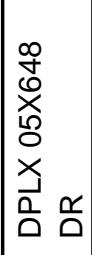 & 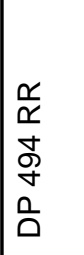 & 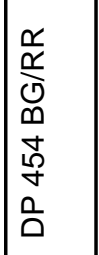 & 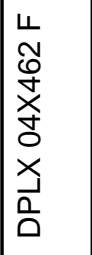 & 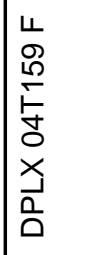 & \\
\hline & 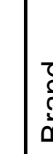 & 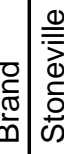 & 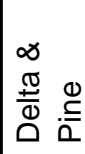 & 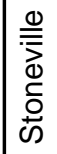 & 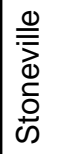 & 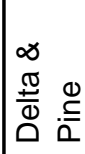 & 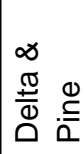 & 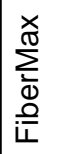 & 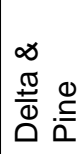 & 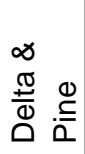 & $\begin{array}{l}\frac{0}{\bar{C}} \\
\frac{d}{0} \\
\frac{0}{\omega} \\
\dot{0}\end{array}$ & 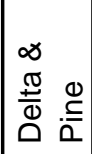 & 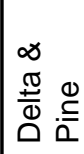 & 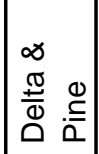 & 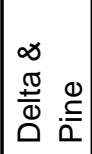 & 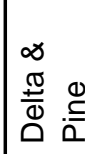 & 믐 \\
\hline
\end{tabular}

